\title{
Calibration of Loop Antennas at VLF
}

\author{
A. G. Jean, H. E. Taggart, and J. R. Wait
}

(February 21, 1961)

\begin{abstract}
A technique and the equipment used for the precise determination of field strength of signals received from VLF transmitters is described. The equipment, which is batteryoperated, contains provisions for the reception of VLF signals and the generation of standard fields to an accuracy of 5 percent. Both the receiving and transmitting antennas are loops. The field strength is determined in terms of a quasi-static magnetic field with the two loop antennas positioned coaxially at a spacing of approximately two meters. Although the technique was developed for use at VLF, it can be used at higher frequencies for calibrating loop antennas, generators, and voltmeters, and for determining effective heights of antennas, or similar applications.
\end{abstract}

\section{Introduction}

With the increased use of the very low frequency portion of the radio spectrum, an urgent need exists for the accurate calibration of receiving antennas operating in this frequency range. Conventional methods of calibrating loop antennas require that they be placed in a radiation field. In other words, the separation between the test source and the receiving antenna is large compared to the wavelength. At VLF this requirement becomes impractical; for example, the wavelength at $10 \mathrm{kc} / \mathrm{s}$ is 30 $\mathrm{km}$. A test source placed at distances greater than this may require several kilowatts of power to provide a signal that will compete with the atmospheric noise level.

It is suggested by Greene [1951] that a free space radiation field could be simulated adequately at the receiving antenna if the test source were also a loop antenna and placed coaxially with, and close to, the receiving loop antenna which is to be calibrated. The magnitude of the equivalent radiation field at the receiving loop antenna is then calculated in terms of the geometry and configuration of the two loops. Such an equation has been stated by Greene [1951] without proof.

It is the purpose of this paper to investigate the general applicability of Greene's equation, to outline a technique used for the precise and accurate determination of field strength using this equation, and finally to describe the equipment used to perform these measurements.

\section{Theoretical Basis}

The necessary equations for calibrating a loop receiving antenna with a coaxial test transmitting loop are first considered. The distance of separation is arbitrary, and the dimensions of the loops are assumed small compared to the wavelength. The situation is illustrated in figure 1.

\footnotetext{
1 Contribution from Central Radio Propagation Laboratory and Radio Stand-
} ards Laboratory, National Bureau of Standards, Boulder, Colo.

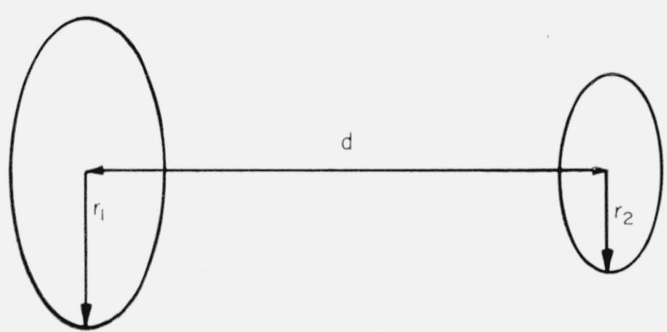

Figure 1. Simplified view of two coaxial loops.

The actual value of the voltage $v$ induced in the receiving loop may be expressed in terms of the maximum equivalent electric field, $E_{f}$, that would exist in a free-space radiation field. The relationship for an electrically small loop is simply

$$
v=j \beta \pi r_{2}^{2} E_{f}, \quad \text { where } \beta=\frac{2 \pi}{\lambda},
$$

but $v=I Z_{m}$, where $Z_{m}$ is the mutual impedance between the loops. Where all dimensions are electrically small and currents in the loop are constant, we have exactly

$$
\begin{aligned}
\mathrm{Z}_{m} & =\left(2 \pi r_{2}\right) \frac{j \mu \omega}{\pi k}\left(\frac{r_{1}}{r_{2}}\right)^{\frac{1}{2}}\left[\left(1-\frac{k^{2}}{2}\right) K(k)-E(k)\right] \\
& =j \mu \omega \frac{\pi r_{2}}{16}\left(\frac{r_{1}}{r_{2}}\right)^{\frac{1}{2}} \times k^{3}\left(1+\frac{3}{4} k^{2}+\frac{75}{128} k^{4}+\ldots\right),
\end{aligned}
$$

where $k=\left[\frac{4 r_{1} r_{2}}{\left(r_{1}+r_{2}\right)^{2}+d^{2}}\right]^{\frac{1}{2}}$ and $r_{1}$ and $r_{2}$ are the transmitting and receiving loop radii, respectively, and $d$ is their center-to-center separation. $K(k)$ and $E(k)$ are elliptic integrals as tabulated by Dwight [1947]. The above equation for $Z_{m}$ may be obtained from a number of different sources, including Smythe [1950] who gives a formula for the $\phi$-component of the 
vector potential, $A_{\phi}$, of a loop carrying a constant current $I$. The mutual impedance $Z_{m}$ is then calculated by integrating $E_{\phi}=-j \omega A_{\phi}$ around the receiving loop. The length of this path is $2 \pi r_{2}$.

Thus,

$$
E_{f} \cong \frac{60 \pi r^{2} I}{\left[d^{2}+\left(r_{1}+r_{2}\right)^{2}\right]^{3 / 2}} \times\left[1+\frac{3}{4} k^{2}+\frac{75}{128} k^{4}+\ldots\right] .
$$

Neglecting terms in the series above $k^{2}$, it is seen that this expression can be reduced as follows

$$
\begin{aligned}
\frac{1+\frac{3}{4} k^{2}}{\left[d^{2}+\left(r_{1}+r_{2}\right)^{2}\right]^{3 / 2}} \simeq \frac{1+3 \frac{r_{1} r_{2}}{d^{2}}+\text { terms in }\left(\frac{r_{1} r_{2}}{d^{2}}\right)^{2}}{\left[d^{2}+r_{1}^{2}+r_{2}^{2}+2 r_{1} r_{2}\right]^{3 / 2}} \\
\simeq \frac{1}{\left[d^{2}+r_{1}^{2}+r_{2}^{2}\right]^{3 / 2}} \times\left[1+\text { terms in }\left(\frac{r_{1} r_{2}}{d^{2}}\right)^{2}\right] .
\end{aligned}
$$

The last expression is the denominator in Greene's equation [1951]. If both loops have radii comparable to $d$, the quantity $k$ is not small compared to unity, and higher terms must be retained in the series. At higher frequencies where $d / \lambda$ cannot be neglected compared to unity, a correction factor may be applied which is the ratio of the coaxial magnetic field of a small loop at wavelength $\lambda$ to the infinite wavelength case. This is known to be $1+j 2 \pi d / \lambda$ and has a magnitude

$$
\left[1+\left(\frac{2 \pi d}{\lambda}\right)^{2}\right]^{\frac{1}{2}}
$$

as stated by Greene [1951]. It is strictly valid for d) $>r_{1}$, but it is only a minor second order correction at medium frequencies and is completely negligible at VLF.

Equation (4) is valid for determining the equivalent free-space electric field strength if $r_{1}, r_{2}$, and $d$ are electrically small compared to $\lambda$. Since this equation is strictly valid only in free-space, the effects of neighboring objects have been verified experimentally by moving metallic objects of various sizes near the loop antennas and observing the effects on the system. If the nearest sizable objects are separated from the loops by approximately two to three times the loop spacing, $d$, the resulting error will be less than about 1 percent. The measurements should not be performed in a shielded room, and the measurement site should be free of large metallic objects such as overhead power lines or similar objects.

\section{Calibration Procedure}

Two basic methods are applicable to the measurenent of field intensity [IRE Standards, 1942]; one is the standard-antenna method, and the other is the standard-field method. In the former a standard receiving antenna is used so that the voltage produced in it by a field of given intensity and polarization may be computed. The usual voltage measured is not the open-circuit antenna voltage but is related to it through a voltage transfer ratio. It is sometimes difficult to determine this voltage transfer ratio to a high degree of accuracy.

In the standard-field method, a standard field is set up by a local transmitting arrangement, and the field at the receiving antenna is computed from the dimensions of the receiving and transmitting antennas, the separation distance between the antennas, and the current in the transmitting loop. In this technique the equipment consists of a suitable receiver equipped with a meter to indicate the relative strength of the received field. The strength of the received field is determined by comparing the receiver output voltage produced by the field under measurement with the voltage produced by a standard field.

Each of the basic methods offers certain advantages and disadvantages dependent upon the application. In the case under consideration, the standard-field method was adopted because of the straightforward calibration method which can be evolved using coaxial loop antenna arrangements as discussed above.

The calibration equipment is represented in figure 2 ; that to the left is used to receive and indicate the relative amplitudes of fields, while that to the right is used to produce VLF fields of appropriate frequency and amplitude. In calibrating, the deflection of the receiver output meter in response to the unknown VLF field is obtained with the receiving loop in the plane of propagation. The receiving loop is then rotated $90^{\circ}$ to minimize the receiver response to this field. The transmitting loop is positioned coaxially with the receiving antenna at a separation distance of 1 or $2 \mathrm{~m}$. Without changing the receiver tuning or sensitivity, the frequency and output of the signal generator are adjusted to reproduce the same receiver output meter indication that was obtained in response to the unknown VLF field. The equivalent value of the radiation field incident at the receiving antenna is calculated from eq (4) above in terms of the antenna radii, their separation distance, and the RF current in the transmitting antenna. The strength of the unknown field is equal to the equivalent radiation component of the locally generated field.

In figure 2, the "Metering Equipment" block is symbolic of the method used to determine the RF current in the transmitting loop. The technique is represented in detail in figure 3 . Balanced RF voltages, obtained from the RF signal generator and Balun (balanced-to-unbalanced transformer), are

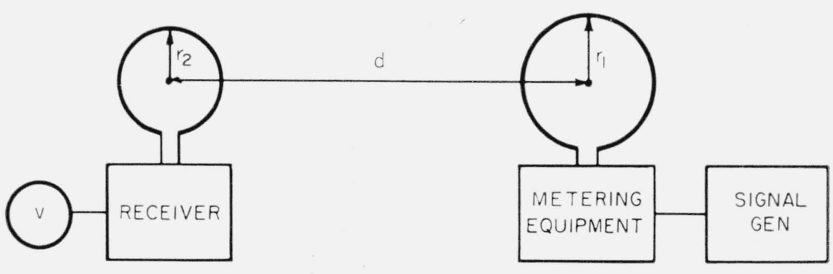

Figure 2. Block diagram of the loop antenna calibration system. 


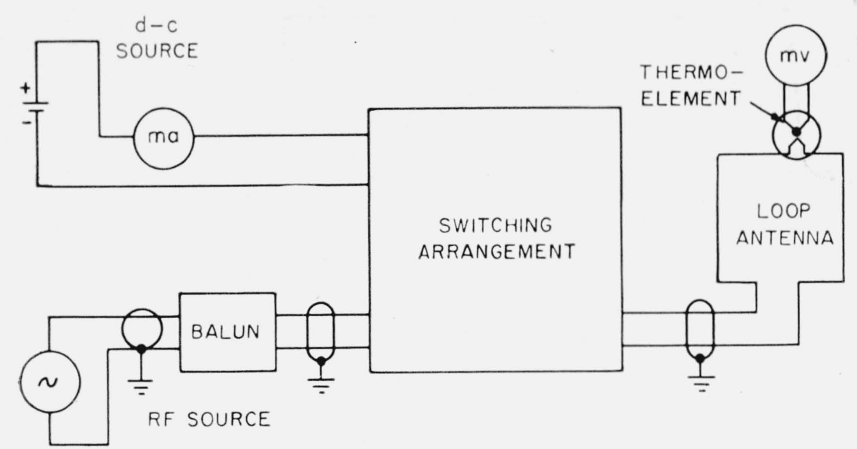

Figure 3. Calibration of the transmitting loop antenna.

connected to the single-turn unshielded transmitting loop. A vacuum thermoelement is mounted in the top-center of the transmitting loop as indicated. The RF current introduced into the loop flows through the thermocouple heater element. The d-c output voltage of the thermoelement is indicated by the $\mathrm{d}$-c millivoltmeter. The RF current is then disconnected from the loop antenna and replaced by a direct current. The direct current is adjusted to produce the same thermoelement d-c output voltage that was attained in response to the RF current. Since equal values of rms RF current and direct current produce equal thermoelement output voltages, the value of direct current read from the milliammeter, figure 3 , is used in eq (4) in calculating the field strength at the receiving loop antenna.

An RF micropotentiometer ${ }^{2}$ [Selby, 1953] was incorporated into the calibration equipment to provide an accurate source of $\mathrm{RF}$ voltages. The arrangement used for calibrating the micropotentiometer and establishing known RF voltages is shown in figure 4 . The thermoelement of the micropotentiometer is the same type used in the transmitting loop. The switching arrangement indicated permits either RF or direct current to flow through the thermoelement and the micropotentiometer resistor element. To establish an accurately known RF voltage across the micropotentiometer, the current required to produce the desired voltage is calculated. A direct current of this magnitude (as indicated by the milliammeter) is caused to flow through the thermocouple and resistor element of the micropotentiometer. The d-c output of the thermoelement in response to the direct current is observed. An RF current of appropriate frequency is then substituted for the direct current and adjusted in magnitude to produce the same thermoelement output voltage that was obtained in response to the direct current. The required RF voltage then exists across the resistive element of the micropotentiometer. The micropotentiometer, having an internal impedance of a few milliohms, is usually used to calibrate equipment having input impedances ranging from 50 ohms upwards. In such cases, the standard voltage across the micropotentiometer is not significantly altered when the equipment under calibration is connected.

${ }_{2}$ This is simply a low-impedance source of RF voltage adjustable from 1 to $10^{5} \mu \mathrm{V}$

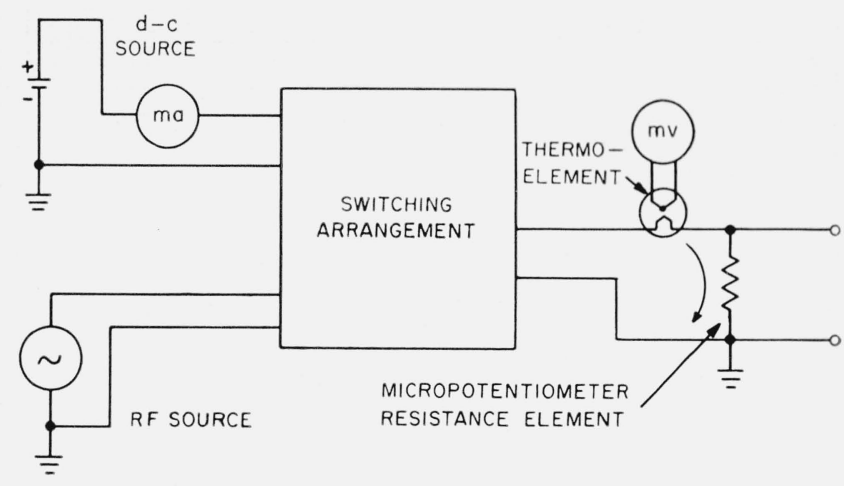

Figure 4. Calibration of the micropotentiometer.

\section{Description of Equipment}

A photograph of the prototype of the field intensity and calibration equipment is given in figure $5 . \mathrm{As}$ viewed from left to right, the individual items are: The receiving loop antenna shown connected by a cable to a box containing a balanced-to-unbalanced electrostatically shielded transformer. The third unit from the left is a transistorized VLF receiver. The next item is a commercially made transistorized VLF signal generator. The transmitting loop antenna is shown mounted on a supporting pedestal with the input terminals near the bottom and the thermoelement and its output at the top. The small box in front and to the right of the transmitting loop contains a transistorized final amplifier for the signal generator. The large box at the extreme right is the calibration unit containing the laboratory type d-c milliammeter and d-c millivoltmeter, the micropotentiometer, switching circuits, etc. A photograph of a collapsible frame to support the transmitting loop antenna (to the left) and the receiving antenna (to the right) is shown in figure 6 . The spacing between the loop antennas in this photograph is $2 \mathrm{~m}$. The framework is made of short sections of tubing which may be disassembled into a "bundle of sticks" for easy transport.

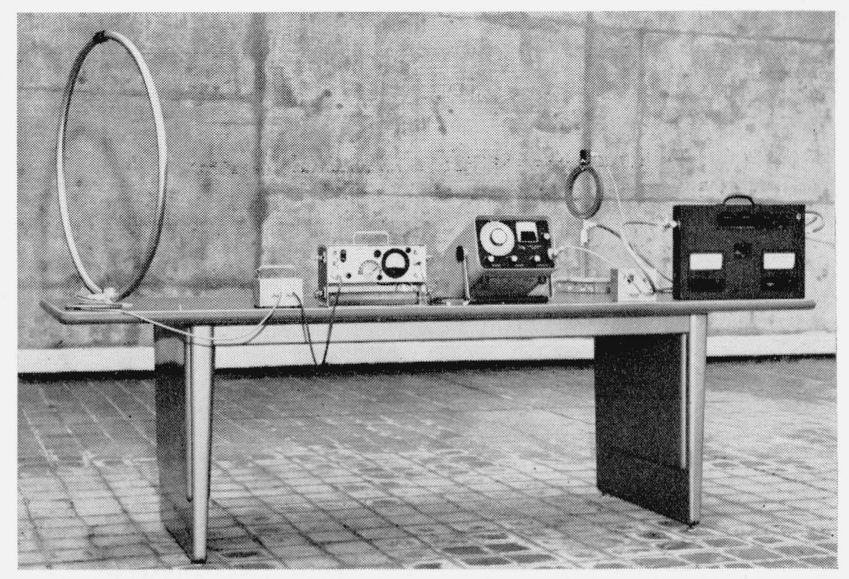

Figure 5. Field intensity and calibration equipment. 


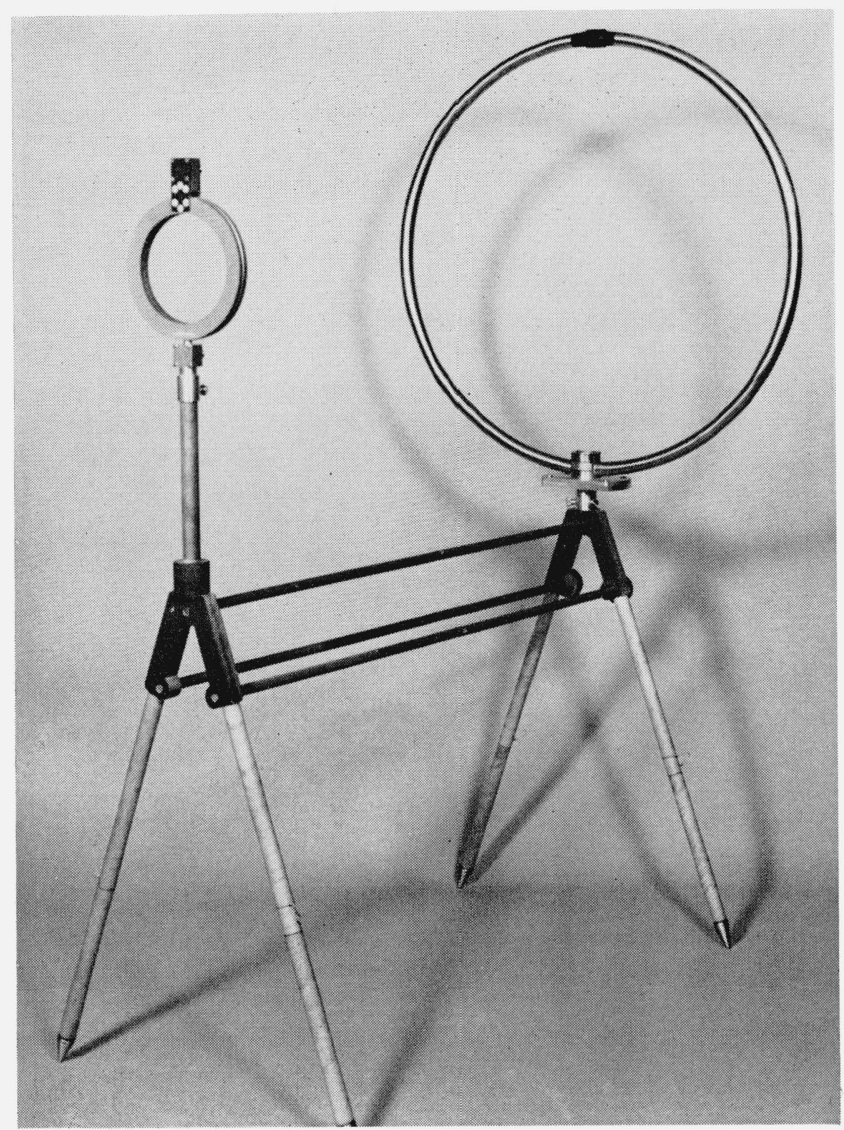

FIGURE 6. Loop antennas mounted on a collapsible frame for field use.

Although all of the equipment shown is batteryoperated and has been used in field work, no serious effort was made to minimize the size or weight of this prototype equipment. Obvious improvements could be made, such as reducing the diameter of the receiving loop and the input transformer, positioning the final amplifier within the signal generator, miniaturizing the calibration unit, etc.

The receiving loop antenna is electrostatically shielded and balanced with respect to ground. The input transformer is a balanced-to-unbalanced unit with an electrostatic shield between the primary and secondary windings. A high degree of balance was achieved in the loop input circuit to minimize undesirable unbalanced currents.

The transistorized, battery-operated VLF receiver shown in the photograph is an early model developed at NBS. An improved model has since been developed which has a sensitivity of about $100 \mu \mathrm{v} / \mathrm{m}$ and a bandwidth adjustable from 40 to $400 \mathrm{c} / \mathrm{s}$. The receiver is tunable from $14.5 \mathrm{kc} / \mathrm{s}$ to $22 \mathrm{kc} / \mathrm{s}$ and is provided with a voltmeter to indicate the relative amplitude of received signals. In the experiment cited, voltmeter readings were made during intervals when station NLK was transmitting a steady carrier signal. It is intended to describe this receiver in a future publication.

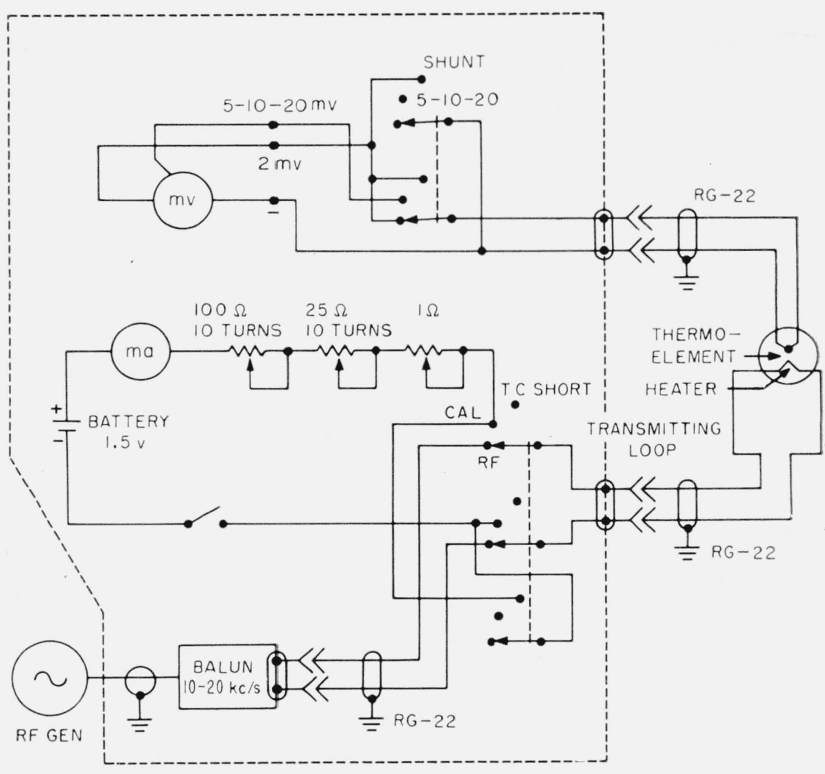

FiguRE 7. Schematic of calibration unit.

A schematic diagram of the calibration unit is shown in figure 7 . The apparatus for the generation of standard fields (represented in fig. 3) and for the calibration of the micropotentiometer (represented in fig. 4) are combined in this unit. The d-c milliammeter and millivoltmeter are high quality laboratory instruments with accuracies of 0.5 percent. Thermoelements of 5- to 100-ma capacity were used in the micropotentiometers, which enabled standard voltages to be generated within a range of 100 to 50,000 $\mu \mathrm{V}$ with an accuracy of better than 3 percent. This type of thermoelement when calibrated with direct current can be used as a current-measuring device up to $300 \mathrm{Mc} / \mathrm{s}$ to an accuracy within 1 percent.

The transmitting loop consists of a single-turn unshielded loop with a 100 ma thermoelement mounted in the top-center. The balun transformer has a voltage unblance that is less than 2 percent of the balanced output voltage, and an insertion loss that is less than $0.3 \mathrm{db}$. This system can be used to establish standard fields with excellent precision and an accuracy that is better than 5 percent.

The transmitting and receiving loop antennas, with radii of approxima tely 0.1 and $0.35 \mathrm{~m}$, respectively, were spaced $2.0 \mathrm{~m}$ from each other. Under these conditions the bracketed series in eq (4) yields a correction factor of approximately 1.028. This represents an increase of approximately 3 percent over the value of the field if the correction is neglected.

The equipment described in this paper was designed to measure field strengths in the range from 230 to $23,000 \mu \mathrm{v} / \mathrm{m}$ at frequencies between 15 and $20 \mathrm{kc} / \mathrm{s}$. The range of frequency coverage can be extended by proper selection of the balun transformer, the signal generator, and the receiver. The micropotentiometer can be used over a voltage range of $1 \mu \mathrm{v}$ to $200 \mathrm{mv}$ at frequencies up to $300 \mathrm{Mc} / \mathrm{s}$. 
The ranges of operation of this equipment can be extended in frequency and amplitude for use in other applications. Excellent precision is obtained with this system with an accuracy not before attainable. All calibrations are performed with direct current. This eliminates many sources of error, and the d-c measuring instruments can readily be checked against reliable d-c working standards. This unit was recalibrated after an extensive field trip, and the calibration of the instruments did not vary more than 0.2 percent.

\section{References}

Dwight, H. B., Tables of integrals and other mathematical data, Revised edition (MacMillan Co., New York, N.Y., 1947).

Greene, F. M., Calibration of commercial field strength meters at the National Bureau of Standards, NBS Circ. 517 (Dec. 1951).

IRE Standards on radio-wave propagation, Measuring Methods (1942).

Selby, M. C., R. F. Micropotentiometers, Radio-Electronic Engineering (Oct. 1953).

Smythe, W. R., Static and dynamic electricity (McGraw-Hill Book Co., Inc., New York, N.Y., 1950).

(Paper 65C3-70) 